\title{
Tabaquismo y enfermedad pulmonar obstructiva crónica: determinación de fracciones atribuibles
}

\author{
H ugo Amigo $C^{1}$, Marcia Erazo $B^{2}$, Manuel 0 yarzún $\mathbf{G}^{3}$, \\ Sergio Bello $S^{4}$, Armando Peruga U 5 . \\ Smoking and chronic obstructive \\ pulmonary disease: attributable risk \\ determination
}

\begin{abstract}
Background: Smoking is the main risk factor for Chronic Obstructive Pulmonary Disease (COPD), an important cause of morbidity and mortality. Aim: To estimate smoking attributable risk and population attributable risk in COPD patients attended in Public Health Services of Santiago. Materials and methods: A case control study matched by sex and age was carried out. Crude and adjusted attributable risks as well as population attributable risk were estimated, controlled by potential confounders and by interaction variables. Results: Mean ages for cases and controls were 68 and 67 years respectively. When compared to the control group, COPD patients had a higher smoking prevalence (at least 100 cigarettes in their life span: $89.7 \%$ vs $60.3 \%$; $<<0.01$ ). Among COPD patients, heavy smokers proportion was 4 times higher than in controls, they smoked for more years ( 43 vs $31 ; p<0.01$ ) and more cigarettes per day ( 18 vs $5 ; \mathrm{p}<0.01$ ). Adjusted attributable risk was 87\% (95\% Confidence Interval (CI): 63.7-94.8). If a patient smoked at least 100 cigarettes in his/her life span and this risk was $92.7 \%$ (CI: 82.4-96.9) for heavy smokers. Projecting this index to Santiago inhabitants, about 87,000 individuals older than 40 years would be suffering COPD due to smoking. Conclusions: This article confirms the strong association between smoking and COPD. Attributable risks are high and significant, even when they are adjusted by confounding variables. Women had a higher risk than men, at lower levels of tobacco consumption (Rev Méd Chile 2006; 134: -1275 82).
\end{abstract}

(Key w ords: Pulmonary disease, chronic obstructive; Smoking, Tobacco)

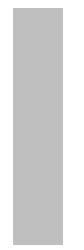

\begin{abstract}
Recibido el 12 de septiembre, 2005. Aceptado el 20 de abril, 2006.
Financiado por la Organización Panamericana de la Salud.

${ }^{1}$ Facultad de Medicina, Universidad de Chile. ${ }^{2}$ Programa de Doctorado en Salud Pública, Escuela de Salud Pública, Universidad de Chile. ${ }^{3}$ Instituto de Ciencias Biomédicas, Facultad de Medicina, Universidad de Chile. ${ }^{4}$ Servicio Médico-Quirúrgico Respiratorio, Instituto Nacional del Tórax. ${ }^{5}$ Programa Regional de Control de Tabaco, Organización Panamericana de la Salud (OPS).
\end{abstract}

Correspondencia a: Dr. Hugo Amigo C. Facultad de Medicina, Universidad de Chile. Avda. Independencia 1027, Santiago. Fono: 56-2-9786213. Fax: 56-2-7355581.

E mail: hamigo@med.uchile 
L a enfermedad pulmonar obstructiva crónica (EPOC) es una causa importante de morbilidad y mortalidad por enfermedad crónica a nivel mundial. La Organización Mundial de la Salud la señaló como la quinta causa de muerte en el mundo y se espera que en las próximas décadas aumente su prevalencia e incidencia1,2. En los últimos 30 años la mortalidad mundial por EPOC ha aumentado $163 \%{ }^{3}$. También representa una carga económica y social, debido a que la morbilidad y la discapacidad asociada a ella, es muchas veces subestimada por pacientes y proveedores de cuidados de salud ${ }^{4}$. En el Reino Unido, se pierden aproximadamente 20 millones de días al año por esta enfermedad ${ }^{5}$ y se espera que sea uno de los factores más comunes de discapacidad en el mundo hacia el año $2020^{6}$.

En Chile, las enfermedades respiratorias son la tercera causa de mortalidad en la población general, y de ellas, la EPOC representa 22\%, siendo la segunda causa de deceso ${ }^{7}$. Anualmente, fallecen entre 1.500 y 1.700 personas por EPOC y en la mayoría de ellas, la muerte ocurre sobre los 65 años de edad, lo que representa una tasa entre 14,9 y 16,9 por 100.000 habitantes mayores de 14

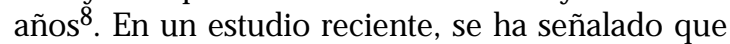
la prevalencia de EPOC en Santiago es 6,3\% de la población mayor de 40 años ${ }^{9}$.

Entre sus factores de riesgo se encuentran hábitos personales y exposición a contaminantes en el domicilio y laborales, pero de todos ellos, el mayor es el tabaquismo, observándose que la prevalencia de la enfermedad en distintos países está relacionada al consumo de tabaco de su población y a la edad de inicio del hábito 4 .

Se estima que alrededor de $30 \%$ de la población mayor de 15 años consume cigarrillos, en el mundo. En América Latina, esta prevalencia es de $26 \% 10$. Varias publicaciones señalan que Chile, históricamente, ha tenido altas prevalencias ${ }^{11-13}$. Estudios realizados últimamente indican que esta frecuencia se ha mantenido estable, con una prevalencia superior a $40 \%$. Se debe también mencionar que se ha comprobado un alarmante consumo en los adolescentes y adultos jóvenes, en quienes la prevalencia sería superior a 55\%, observándose además, un incremento de este hábito en las mujeres y una disminución en la edad de inicio de su consumo ${ }^{14}$.
La asociación entre tabaquismo y EPOC ha sido documentada previamente. Sin embargo, el cálculo de su fracción atribuible y cuantificación poblacional ha sido menor. Este estudio, que se enmarca en un proyecto multicéntrico que se realiza en cuatro ciudades de América Latina (Bogotá, Ciudad de México, Río de Janeiro y Santiago), cuyo propósito es estimar los costos económicos derivados de la atención médica en patologías atribuibles al consumo de tabaco y es coordinado por la Organización Panamericana de la Salud (OPS). Este trabajo está destinado específicamente a estimar el impacto del consumo de tabaco sobre la ocurrencia de EPOC en la población mayor de 40 años de Santiago, Chile.

\section{MATERIAL Y MÉTODO}

Para estimar la fracción atribuible se diseñó un estudio de casos y controles pareado por edad y sexo. Se consideró caso a aquel individuo con diagnóstico clínico de enfermedad pulmonar obstructiva crónica ( proceso patológico que se caracteriza por una limitación del flujo aéreo que no es completamente reversible» GOLD $2001^{15}$ que según la clasificación internacional corresponde a los códigos CIE-10 J44.8-J44.9 que incluye bronquitis crónica y enfisema pulmonar. Además los pacientes que ingresaron al estudio no tenían una fecha de diagnóstico superior a dos años. Ninguno de los casos incluidos en este estudio presentó EPOC grave, ya que no tuvieron $\mathrm{VEF}_{1}<30 \%$, ni $\mathrm{VEF}_{1}<50 \%$ asociado a insuficiencia respiratoria 0 insuficiencia cardíaca derecha, según lo que constaba en los antecedentes espirométricos y el diagnóstico clínico. Los pacientes EPOC fueron identificados y entrevistados entre 2001 y 2003 en el Instituto Nacional del Tórax y en Centros de Atención Primaria de Salud, de Santiago. Para cada paciente «caso», en los 15 días posteriores a su reclutamiento, se seleccionaron, en forma aleatoria, de entre los individuos que solicitaban atención ambulatoria que no requerían internación, dos controles de igual sexo y edad $( \pm 3$ años) de los servicios ambulatorios de oftalmología, otorrinolaringología, traumatología y cirugía de hospitales base y de centros de atención primaria de salud. No fueron incorporados al estudio individuos-casos que presentaban antece- 
dentes de tuberculosis, silicosis; cáncer de cualquier tipo, asma u otra enfermedad respiratoria.

El tamaño muestral fue de 44 mujeres con EPOC y 88 controles de la misma edad ( \pm 3 años), el que fue calculado considerando $32 \%$ de prevalencia de tabaquismo y una fracción atribuible esperada de 0,50. En el caso de los hombres, ésta fue de 43 casos y 86 controles de igual edad $( \pm 3$ años), considerando $40 \%$ de tabaquismo y una fracción atribuible esperada de 0,60. En ambos cálculos, se consideró un alfa de 0,025 y un poder del test de $80 \%$. El cálculo fue realizado según la fórmula de Browner y Newman ${ }^{16}$.

La información fue recolectada en cuestionarios especialmente diseñados para este fin por especialistas de los cuatro países involucrados en el estudio multicéntrico y de la Organización Panamericana de la Salud, que consideraba información acerca de la historia de tabaquismo de la persona así como potenciales fuentes de contaminación laborales e intradomiciliarias. La recolección fue efectuada por enfermeras capacitadas y permanentemente supervisadas, previa explicación de los objetivos a cada paciente y solicitud de participación que incluyó la firma de un acta de consentimiento informado. El proyecto en Chile fue aprobado por el Comité de Ética de Investigación de la Facultad de Medicina de la Universidad de Chile.

El análisis comprendió las siguientes etapas: Fase exploratoria de los datos, identificando su distribución, valores atípicos y estimando medidas de tendencia central y dispersión. La exposición a tabaquismo se caracterizó de dos maneras: la primera consideró si la persona fumó o no, al menos, cien cigarrillos durante su vida y la segunda definió gran fumador considerando los paquetes/años fumados (número de cigarrillos fumados por día x años de fumador/20) y para definir su punto de corte, se hizo un análisis de la sensibilidad y especificidad (valor más alto del índice de Youden) del tabaco en relación a la EPOC, para cada uno de los sexos en forma independiente. Para hombres, se estableció como gran exposición al tabaco el haber fumado más de 19 paquetes año y para mujeres, el haber fumado más de 9 paquetes año. Se controló por potenciales variables confusoras y de interacción (educación, contaminación ambiental, cocina $\mathrm{y}$ calefacción con leña, carbón o parafina, tabaquis- mo pasivo en domicilio y trabajo, antecedentes familiares de EPOC y exposición laboral a otros contaminantes: asbesto, plomo, sílice, arsénico), las que fueron analizadas en forma dicotómica que resume si la conducta o exposición existió o no (ejemplo: fumador pasivo, exposición laboral o domiciliaria a otros contaminantes).

Se estimó la razón de disparidad: OR (Odds Ratio) en forma cruda y ajustada entre las distintas variables de exposición al tabaco y EPOC a través de la construcción de modelos de regresión logística condicional, controlando por posibles efectos de confusión e interacción. Se consideró significativo un valor de $\mathrm{p}<0,05$ para las asociaciones y para las interacciones un valor de $\mathrm{p}<0,1$. Con el fin de estimar la magnitud del impacto del tabaquismo en la EPOC, se calculó el número de pacientes con EPOC atribuibles al tabaquismo. Las prevalencias de EPOC que se utilizaron fueron las comunicadas en el informe del estudio Platino para Chile ${ }^{9}$.

La estimación de la fracción atribuible (FA) se realizó de acuerdo a la siguiente fórmula ${ }^{16}$.

$$
\mathrm{FA}=\frac{(\mathrm{OR}-1)}{\mathrm{OR}}
$$

Y para la fracción atribuible poblacional (FAP) ${ }^{18}$

$$
\mathrm{FAP}=\frac{\pi \cdot(\mathrm{OR}-1)}{\mathrm{OR}}
$$

Siendo $\pi$ la proporción de expuestos y OR, odds ratio (razón de disparidad).

La digitación de los datos se realizó en el programa EPI Data versión $2.1^{19}$, efectuándose doble digitación. El procesamiento de la información se hizo utilizando el paquete estadístico SPSS versión $12.0^{20}$ y el análisis en el Programa Stata versión $8.0^{21}$.

\section{RESULTADOS}

La mediana de edad de las personas entrevistadas fue de 68 años para casos y controles, siendo las mujeres tres años menores. La mediana de años de escolaridad fue de ocho años para los casos y de un año menos para los controles y, al comparar las diferencias entre sexos, las mujeres presentaron dos años menos de escolaridad (Tabla 1). Destaca que los pacientes con EPOC tuvieron una 
mayor exposición al tabaquismo (casi 30\% más), la proporción de grandes fumadores fue casi 4 veces mayor, fumaron significativamente más años (12 años más; $\mathrm{p}<0,01$ ) y tendieron a consumir grandes cantidades diarias de cigarrillo $(p<0,01)$, en comparación con los controles, tendencia que fue similar en ambos sexos (Tabla 1).

El riesgo de tener EPOC en aquellos individuos que fumaron, al menos, cien cigarrillos en la vida, fue alto en los dos sexos, aunque algo menor en los varones. Cuando se calcularon los Odds ratio en aquellos individuos que eran grandes fumadores, éstos fueron muy altos en los hombres y menores en las mujeres. Al ajustar por potenciales variables confundentes, el riesgo en los hombres disminuyó, situación opuesta a lo observado en las mujeres (Tabla 2). Es importante mencionar que no se encontraron valores atípicos ni interacción en la construcción de los modelos.

Se estimó la fracción atribuible al tabaco, observándose un comportamiento diferenciado de acuerdo a la definición de exposición a tabaquismo, cuando se consideran expuestos los que han fumado al menos cien cigarrillos. En hombres y mujeres alcanzó $85 \%$ y se considera gran fumador, la FA aumentó en ambos sexos, siendo levemente mayor en hombres (Tabla 2). Considerando las distintas definiciones de exposición a tabaco de todos los pacientes con EPOC de la muestra, se estimó la fracción atribuible poblacional, que resultó ser mayor a $74 \%$ en los que fumaron cien cigamillos y $65 \%$ entre los grandes fumadores (Tabla 2).

Finalmente, se calculó la población con EPOC atribuible al consumo de tabaco considerando las fracciones poblacionales del gran fumador. El número de habitantes de Santiago mayor de 40 años con EPOC atribuible al consumo de tabaco llegaría alrededor de 87.000 personas, destacando un mayor número absoluto de hombres, debido a que presentaron una mayor prevalencia de esta enfermedad que las mujeres (Tabla 3).

\section{Discusión}

Este estudio confirma la gran importancia del tabaco en el desarrollo de la enfermedad pulmonar obstructiva crónica. Sus riesgos y fraccio-

Tabla 1. Caracterización del hábito tabáquico y sociodemográfica de la población estudiada

\begin{tabular}{|c|c|c|c|c|c|c|c|c|}
\hline \multirow{3}{*}{ Exposición } & \multicolumn{4}{|c|}{ Frecuencia (\%) } & \multicolumn{4}{|c|}{$\begin{array}{c}\text { Mediana } \\
\text { (Dispersión intercuartílica) }\end{array}$} \\
\hline & \multicolumn{2}{|c|}{ Mujeres } & \multicolumn{2}{|c|}{ Hombres } & \multicolumn{2}{|c|}{ Mujeres } & \multicolumn{2}{|c|}{ Hombres } \\
\hline & $\begin{array}{l}\text { Caso } \\
(\mathrm{n}=44)\end{array}$ & $\begin{array}{l}\text { Control } \\
(\mathrm{n}=88)\end{array}$ & $\begin{array}{l}\text { Caso } \\
(\mathrm{n}=43)\end{array}$ & $\begin{array}{l}\text { Control } \\
(\mathrm{n}=86)\end{array}$ & Caso & Control & Caso & Control \\
\hline Tabaquismo & & & & & & & & \\
\hline Ha fumado en la vida & $87,0 \dagger$ & 50,0 & 92,7 & 72,0 & & & & \\
\hline Gran fumador & $71,7 \dagger$ & 15,9 & $73,2 \dagger$ & 20,7 & & & & \\
\hline Convive con fumador & 69,6 & 69,8 & 75,6 & 51,2 & & & & \\
\hline $\begin{array}{l}\text { Trabaja con personas } \\
\text { que fuman }\end{array}$ & $50,0 \dagger$ & 23,9 & $51,2 \dagger$ & 28,0 & & & & \\
\hline & & & & & $17^{*}$ & 20 & 17 & 17 \\
\hline Edad inicio consumo & & & & & $\begin{array}{c}(13-21) \\
40 *\end{array}$ & $\begin{array}{c}(15-25) \\
26\end{array}$ & $\begin{array}{c}(14-20) \\
43 *\end{array}$ & $\begin{array}{c}(15-20) \\
36\end{array}$ \\
\hline Años fumado & & & & & $(31-53)$ & $(15-41)$ & $(35-52)$ & $(25-47)$ \\
\hline & & & & & $10^{*}$ & 4 & $20^{*}$ & 5 \\
\hline Número de cigarrillos diarios & & & & & $(6-20)$ & $(2-10)$ & $(10-30)$ & $(1-20)$ \\
\hline Edad (años) & & & & & $\begin{array}{c}65 \\
(57-73)\end{array}$ & $\begin{array}{c}65 \\
(56-73)\end{array}$ & $\begin{array}{c}68 \\
(65-75)\end{array}$ & $\begin{array}{c}68 \\
(63-74)\end{array}$ \\
\hline Escolaridad (años estudio) & & & & & $\begin{array}{c}7 \\
(4-12)\end{array}$ & $\begin{array}{c}6 \\
(3-10)\end{array}$ & $\begin{array}{c}9 \\
(5-12)\end{array}$ & $\begin{array}{c}8 \\
(4-12)\end{array}$ \\
\hline
\end{tabular}

tp $<0,05$ en $\mathrm{X}^{2 *} \mathrm{p}<0,05$ en Test de Mediana. 
nes atribuibles son altas y significativas, indistintamente de la definición de exposición adoptada, situación que se mantiene al ajustar por potenciales factores confundentes. Además, se observa un comportamiento diferenciado por sexo, en que las mujeres presentan valores altos con una intensidad menor de consumo de tabaco.

Tabla 2. Riesgos, fracciones atribuibles y fracciones atribuibles poblacionales crudos y ajustados de presentar EPOC de acuerdo a distintos niveles de exposición de tabaquismo

\begin{tabular}{|c|c|c|c|c|c|}
\hline \multirow[t]{2}{*}{ Conducta } & \multirow[t]{2}{*}{$\begin{array}{l}\text { Medida de } \\
\text { asociación }\end{array}$} & \multicolumn{2}{|c|}{$\begin{array}{l}\text { Valor crudo } \\
\text { (IC 95\%) }\end{array}$} & \multicolumn{2}{|c|}{$\begin{array}{l}\text { Valor ajustado* } \\
\text { (IC 95\%) }\end{array}$} \\
\hline & & Mujeres & Hombres & Mujeres & Hombres \\
\hline Haber fumado & OR & $\begin{array}{c}7,14 \\
(2,44-20,88)\end{array}$ & $\begin{array}{c}6,73 \\
(1,50-30,05)\end{array}$ & $\begin{array}{c}7,88 \\
(2,55-24,36)\end{array}$ & $\begin{array}{c}4,65 \\
(0,98-22,09)\end{array}$ \\
\hline Gran fumador & OR & $\begin{array}{c}11,4 \\
(3,9-32,8)\end{array}$ & $\begin{array}{c}18,01 \\
(4,22-76,83)\end{array}$ & $\begin{array}{c}13,65 \\
(4,2-44,9)\end{array}$ & $\begin{array}{c}14,97 \\
(3,02-74,1)\end{array}$ \\
\hline Haber fumado & FA & $\begin{array}{c}85,9 \\
(59,0-95,2)\end{array}$ & $\begin{array}{c}85,1 \\
(33,3-96,6)\end{array}$ & $\begin{array}{c}87,3 \\
(60,7-95,8)\end{array}$ & $\begin{array}{c}78,5 \\
(2,0-95,4)\end{array}$ \\
\hline Gran fumador & FA & $\begin{array}{c}91,2 \\
(74,3-96,9)\end{array}$ & $\begin{array}{c}94,4 \\
(76,3-98,7)\end{array}$ & $\begin{array}{c}92,6 \\
(76,2-97,8)\end{array}$ & $\begin{array}{c}93,2 \\
(66,9-98,6)\end{array}$ \\
\hline Haber fumado & FAP & $\begin{array}{c}74,7 \\
(51,3-82,8)\end{array}$ & $\begin{array}{c}78,9 \\
(30,8-89,8)\end{array}$ & $\begin{array}{c}75,9 \\
(52,8-83,3)\end{array}$ & $\begin{array}{c}72,7 \\
(1,8-88,4)\end{array}$ \\
\hline Gran fumador & FAP & $\begin{array}{c}65,4 \\
(53,3-69,5)\end{array}$ & $\begin{array}{c}69 \\
(55,8-72,2)\end{array}$ & $\begin{array}{c}66,4 \\
(54,7-70,2)\end{array}$ & $\begin{array}{c}68,2 \\
(4,89-72,1)\end{array}$ \\
\hline
\end{tabular}

*Ajustado = Ajustado por educación, contaminación ambiental (cocina y calefacción) con leña, carbón y parafina, tabaquismo pasivo en domicilio y trabajo, antecedentes familiares de EPOC y exposición laboral a otros contaminantes (asbesto, plomo, sílice, arsénico).

IC 95\%= Intervalo de Confianza de 95\% del OR; FA; FAP.

$\mathrm{OR}=$ Odds Ratio

$\mathrm{FA}=$ Fracción atribuible.

$\mathrm{FAP}=$ Fracción atribuible poblacional.

Tabla 3. Proyección de población con EPOC mayor de 40 años atribuible al tabaco en Santiago. Exposición gran fumador

\begin{tabular}{|lccccc|}
\hline Población & Población total $†$ & $\begin{array}{c}\text { Prevalencia } \\
\text { de EPOC* } \\
\%\end{array}$ & $\begin{array}{c}\text { Población con } \\
\text { EPOC }\end{array}$ & $\begin{array}{c}\text { Fracción atribuible } \\
\text { poblacional } \\
\text { (Intervalo de confianza) }\end{array}$ & $\begin{array}{c}\text { Población con EPOC } \\
\text { atribuible al tabaquismo } \\
\text { (Intervalo de confianza) }\end{array}$ \\
\hline Mujeres & 1.120 .555 & 5,6 & 62.751 & $\begin{array}{c}66,4 \\
(54,7-70,2)\end{array}$ & $\begin{array}{c}41.666 \\
68,2\end{array}$ \\
Hombres & 936.051 & 7,5 & 70.203 & $(4,89-72,1)$ & $47.47 .051)$ \\
Total & 2.056 .606 & 6,3 & 129.566 & 67,1 & $(3.433-50.616)$ \\
& & & & $(59,7-70,2)$ & 86.939 \\
& & & & & \\
\hline
\end{tabular}

†Censo 2002.

*Prevalencia reportada por referencia 9. 
Si bien, la definición de tabaquismo no afecta la directa asociación con EPOC, ésta sí tiene plena relevancia al momento de estimar la fuerza de la asociación según sexo. En este estudio, se observó que cuando la definición considera un menor consumo de tabaco, las mujeres aparecen con riesgos más altos que los hombres $\mathrm{y}$, por el contrario, cuando se optó por una definición que incorporaba mayor cantidad de tabaco consumido, los hombres presentan mayores riesgos que las mujeres. La definición de expuestos es importante, especialmente en un país en que hombres y mujeres presentan una alta prevalencia de tabaquismo, pero con distintos niveles de consumo ${ }^{22}$.

También, e indistintamente de la definición de exposición a tabaquismo, las fracciones atribuibles son altas, lo que ratifica lo planteado por otros estudios que señalan al tabaquismo como el principal factor de riesgo de esta patología 23,24 . Debe destacarse que este riesgo es mayor al comunicado en otras latitudes. Esto es un hecho interesante, dadas las condiciones de contaminación aérea existentes en la ciudad de Santiago y señala la relevancia de considerar las estimaciones de riesgo según las características de cada sitio y no inferirla de valores obtenidos en otros lugares. Por otra parte, al excluir los pacientes con EPOC en etapas avanzadas es posible que la fracción atribuible haya sido atenuada en cierto grado.

Sin embargo, como ha sido planteado previamente, la fuerza de asociación varía en cada sexo, dependiendo de la definición de exposición. Se podría argumentar sobre la asociación en mujeres con una definición menos estricta de tabaquismo, que ellas presentan mayor sintomatología que los hombres a un mismo nivel de consumo de taba$\mathrm{co}^{25,26}$, lo que podría ser explicado, en parte, por un menor calibre de las vías respiratorias de la mujer comparado con las de un hombre de igual estatura, lo cual hace que a un grado similar de aspiración de un cigarrillo que un hombre, gases y partículas alcanzarían una mayor concentración en las vías aéreas de la mujer y, por ello, la respuesta bronquial senía mayor a estos estímulos exógenos ${ }^{27,28}$.

Asimismo, es posible plantear que existiría una interacción entre el hecho de fumar y ser mujer, hecho que no fue comprobado en este estudio, pero que ha sido sugerido en diversas publicaciones. En ellas se ha manifestado que las mujeres serían más susceptibles a desarrollar EPOC, incluso en su forma más avanzada, con menores concentraciones de humo de tabaco ${ }^{29,30}$. Aunque no existe consenso en las investigaciones publicadas $^{31}$. Entre las explicaciones que se han dado a este hecho, se plantea que el tabaco poseería un efecto antiestrogénico que podría explicar las diferencias en mujeres ${ }^{32}$. Esta hipótesis se basa en que, en animales de experimentación, se ha encontrado que los estrógenos poseen la capacidad de regular el tamaño y número de alvéolos, disminuyendo su pérdida e induciendo su regeneración ${ }^{33}$.

Por el contrario, cuando se hizo más estricta la definición de tabaquismo, la mayor asociación se observó en los hombres. Es difícil encontrar una explicación biológicamente plausible para este fenómeno, el cual podría ser explicado por la gran cantidad de cigarrillos que ellos consumen, mayor que el de las mujeres.

El valor de la fracción atribuible poblacional permite estimar el número de posibles personas afectadas con EPOC derivadas del consumo de tabaco, con lo cual se pueden hacer proyecciones sobre la demanda de la atención de salud y de los costos asociados a la enfermedad y en cuánto podńa reducirse si la población dejara de fumar. Es necesario resaltar que el cálculo de fracciones atribuibles y el estudio de prevalencia de EPOC de donde se obtuvieron las cifras presentadas en este artículo fuemon hechos para Santiago, por lo que el cálculo de la población afectada es válido sólo para esta ciudad.

Otro aspecto que es interesante mencionar es que la fracción atribuible encontrada es mayor (20\%) a lo publicado en estudios internacionales, en que los valores oscilan entre $68 \%$ y $73 \% 34,35$. Este hecho ha generado una discusión si estos riesgos atribuibles pueden ser explicados por diferencias en los patrones de consumo del tabaco (marca, inhalación), contaminación ambiental e intradomiciliaria de cada población, hipótesis que en este artículo no es posible comprobar, pero que plantea un importante desafío a investigar 36,37 .

En la inferencia poblacional de estos cálculos, se debe considerar que en este estudio usamos la definición GOLD 2001 para el diagnóstico de EPOC, la cual, además de la razón fija $\mathrm{VEF}_{1} / \mathrm{CVF}$ menor de 0,7, incluye una disminución de $\mathrm{VEF}_{1}$ por debajo de $80 \%$ de su valor predeterminado. Con esta definición la prevalencia de EPOC para Santiago fue de $6,3 \%$ en el estudio PLATINO ${ }^{9}$. Sin 
embargo, en una publicación más reciente de este mismo estudio ${ }^{38}$, se utilizó sólo la razón fija de $\mathrm{VEF}_{1} / \mathrm{CVF}$ menor de 0,7 con lo que se obtuvo una prevalencia mayor de EPOC (16,9\%) que al utilizar el criterio GOLD 2001. Según sus propios autores, el estudio al utilizar solamente la relación fija se podrían incluir falsos positivos. Por ello, todas las estimaciones poblacionales en el presente estudio fueron realizadas considerando una prevalencia de EPOC de 6,3\%. En esta estimación de prevalencia $^{9}$ se incluyeron los casos graves, que en rigor para nuestro estudio, deberían haberse eliminado de los cálculos, ya que fueron excluidos en la selección de casos. Sin embargo, se siguió considerando la prevalencia de 6,3\%, ya que dudamos que los casos graves tengan una fracción atribuible menor y porque corresponden a una proporción muy baja en cualquier población de EPOC.

Una de las limitaciones que pueden señalarse de este estudio es que los controles son de base hospitalaria, por lo que sería posible pensar que no son representativos de la exposición a tabaquismo de la población general ${ }^{39}$. Entre los sesgos de selección descritos que se podrían postular en este estudio, se encuentra el de Berkson, que se produce al existir un aumento

\section{REFERENCIAS}

1. WHO. World Health Report 2002. http://www.w ho.int/whr/2002/en/(consultado: 08.09. 2004).

2. MurRay CJ, López AD. Alternative projections of mortality and disability by cause 1990-2020: Global Burden of Disease Study. Lancet 1997; 349(9064): 1498-504.

3. Gil R, Beшo S. Lanzamiento de GOLD en Chile. Rev Chil Enf Respir 2002; 18: 61-81.

4. Paumels R, Rabe K. Burden and clinical features of chronic obstructive pulmonary disease (COPD). Lancet 2004; 364: 613-20.

5. BARNES P. COPD-a neglected disease. Lancet 2004; 364: 564-5.

6. López AD, Murray CC. The global burden of disease, 1990-2020. Nat Med 1998; 4: 1241-3.

7. Szoт J. Mortalidad por enfermedades respiratonias en Chile durante 1999. Rev Chil Enf Respir 2003; 19: 8-14.

8. Sociedad Chilena de Enfermedades Respiratorias. Consenso Nacional en Enfermedad Pulmonar Obstructiva Crónica (EPOC). Rev Chil Enf Respir 1998; 14: 2. en las tasas de admisión hospitalaria a personas con determinadas exposiciones de interés ${ }^{40}$. En este estudio, si bien los controles fueron seleccionados de aquellos servicios que atienden enfermedades que no están directamente asociadas o relacionadas con el tabaquismo, la alta prevalencia del hábito en estos controles hace pensar que es posible que exista este tipo de sesgo, lo que podría redundar en una disminución espuria del Odds Ratio.

Finalmente, si las autoridades desean disminuir los costos sociales y económicos que la EPOC impone al sector salud y a la sociedad en general, será necesario hacer esfuerzos tendientes a evitar que la población inicie el consumo de tabaco y en aquellos que ya fuman, instarlos a dejar de hacerlo. Es importante resaltar que a nivel mundial, se ha comunicado un aumento en la prevalencia de EPOC en la población general, que obedece a un incremento en el consumo de tabaco ${ }^{41,42}$. Esta situación es preocupante, ya que podría suceder lo mismo en nuestro país, por lo que es necesario que las autoridades adopten rápidamente todas las estrategias necesarias para evitar que más personas enfermen de EPOC u otras enfermedades a causa del tabaquismo.

9. BaptisTa AM, Victora C. Platino Study_Chilean Survey Report. Disponible en: http://www.platinoalat.org/docs/report platino chile 2004.pdf (consultado 05.12. 2005).

10. WHO-IARC Monographs on the evaluation of carcinogenic risks to humans. Vol 83, Tobacco smoke and involuntary smoking. Lyon, France, IARC Press, 2004.

11. Jadue L, Vergara J, Escobar M, Deigado I, Garrido C, LASTRA P ET AL. Factores de riesgo para las enfermedades no trasmisibles: Metodología y resultados globales del programa CARMEN. Rev Méd Chile 1999; 127: 1004-13.

12. Medina E, Kaemprer A, Cornejo E, Hernández E. Tabaquismo en Santiago, 1993-94. Rev Méd Chile 1995; 123: 652-8.

13. Gutiérrez M, Rioseco F, Rojas A, Casanova D, Cordero M, SchiafFino M. Prevalencia de tabaquismo en la población general de Valparaíso y Viña del Mar. Rev Méd Chile 1995; 123: 250-6.

14. Alvarado R, Talavera G. La epidemia de tabaquismo en Chile: Evolución durante la última década. Rev Chil Salud Pública 2003; 7: 69-73. 
15. Pauweis RA, Buist SA, Calveriey PM, Jenkins CR, Hurd SS. Global Strategy for the Diagnosis, Management, and Prevention of Chronic Obstructive Pulmonary Disease (NHLBI/WHO GOLD workshop summary) Am J Respir Crit Care Med 2001; 163: 1257-78.

16. Browner WS, Newman TB. Sample size and power based on the population attributable fraction. Am J Public Health 1989; 79: 1289-94.

17. KuRITZ S, LANDIS JR. Attributable risk estimation from matched case-control data. Biometrics 1988; 44: 355-67.

18. MAC MAhon B, Trichopoulos D. Epidemiología. $2^{\text {a }}$ Ed. Marbán, SL. Madrid, España, 2001.

19. Lauritsen JM, Bruus M, Myatt MA. EpiData, version 2.1. An extended tool for validated entry and documentation of data. The EpiData Asociation, Odense, Denmark. 2001.

20. SPSS para Windows. Versión 12.0.1. Chicago: SPSSInc. 2000.

21. STATACORP. Stata Statistical Software: Release 8.0. College Station, TX: Stata Corporation, 2003.

22. Ministerio de Salud de Chile. Departamento de Epidemiología. Encuesta Nacional de Salud 2003. Disponible en: http://www.minsal.cl (consultado: 24.08.2004)

23. Jaén díaz Ji, De Castro Mesa C, Gontan GarcíaSalamanca MJ, López De Castro F. Prevalence of Chronic Obstructive Pulmonary Disease and Risk Factors in Smokers and Ex-smokers. Arch Bronconeumol 2003; 39: 554-8.

24. Rodríguez Tapioles R, Bueno Cavanilas A, Pueyos Sánchez A, Espigares García M, Martínez González MA, Gálvez VARGas R. Morbidity, mortality and the potential years of life lost attributable to tobacco. Med Clin (Barcelona) 1997; 108: 121-7.

25. LANGhammer A, Johnsen R, Holmen J, Gulsvik A, BJERMER L. Cigarette smoking gives more respiratory symptoms among women than among men. The Nord-Trondelag Health Study (HUNT). J Epidemiol Community Health 2000; 54: 917-22.

26. Langhammer A, Johnsen R, Gulsvik A, Holmen TL, BJERMER L. Sex differences in lung vulnerability to tobacco smoking. Eur Respir J 2003; 21: 1017-23.

27. CHAPMAN KR. Chronic obstructive pulmonary disease: are women more susceptible than men? Clin Chest Med 2004; 25: 331-41.

28. Leynaert B, Bousquet J, Henry C, Luard R, Neukirch F. Is bronchial hyperresponsiveness more frequent in women than in men? A population-based study. Am J Respir Crit Care Med 1997; 156: 1413-20.

29. Prescott E, Bjerg AM, Andersen PK, Lange P, VestBo J. Gender difference in smoking effects on lung function and risk of hospitalization for COPD: results from a Danish longitudinal population study. Eur Respir J 1997; 10: 822-7.

30. Silverman EK, Weiss ST, Drazen JM, Chapman HA, Carey V, Campbell EJ et al. Gender-related differences in severe, early-onset chronic obstructive pulmonary disease. Am J Respir Crit Care Med 2000; 162: 2152-8.

31. TANoue LT. Cigarette smoking and women's respiratory health. Clin Chest Med 2000; 21: 47-65.

32. MeEK MD, Finch GL. Diluted mainstream cigarette smoke condensates activate estrogen receptor and aryl hydrocarbon receptor-mediated gene transcription. Environ Res 1999; 80: 9-17.

33. MASSARO D, MASSARO GD. Estrogen regulates pulmonary alveolar formation, loss, and regeneration in mice. Am J Physiol Lung Cell Mol Physiol 2004; 287: L1154-9.

34. Ruff LK, Volmer T, Nowak D, Meyer A. The economic impact of smoking in Germany. Eur Respir J 2000; 16: 385-90.

35. JohanNessen A, OMenaAs ER, BaKKe PS, GuLSVIK A. Implications of reversibility testing on prevalence and risk factors for chronic obstructive pulmonary disease - a community study. Thorax 2005 published on line 5 Aug 2005; doi: 10.1136/thx.2005.043943.

36. Lange P, Nyboe J, Appleyard M, Jensen G, Schnohr P. Relationship of the type of tobacco and inhalation pattern to pulmonary and total mortality. Eur Respir J 1992; 5: 1111-7.

37. Lange P, Groth S, Nyboe J, Mortensen J, Appleyard M, JeNSEN G ET AL. Decline of the lung function related to the type of tobacco smoked and inhalation. Thorax 1990; 45: 22-6.

38. Menezes AM, Pérez-Padila R, Jardim JR, Muino A, López MV, VaLdivia G et al. Chronic obstructive pulmonary disease in five Latin American cities (the PLATINO study): a prevalence study. Lancet 2005; 366(9500): 1875-81.

39. DAYA S. Characteristics of good causation studies. Semin Reprod Med 2003; 21: 73-83.

40. GRimEs DA, Schulz KF. Bias and causal associations in observational research. Lancet 2002; 359(9302): 248-52.

41. DoherTy DE, Briggs DD JR. Chronic obstructive pulmonary disease: epidemiology, pathogenesis, disease course, and prognosis. Clin Cornerstone 2004; Suppl 2: S5-16.

42. Mannino DM. COPD: epidemiology, prevalence, morbidity and mortality, and disease heterogeneity. Chest 2002; 121(5 Suppl): 121S-126S. 